\title{
Application of similarity analysis method in zero-power experimental valida- tion
}

\author{
Tong Ning ${ }^{1, *}$, Qi Zhou ${ }^{1}$, Xiaoliang Chen $^{1}$, and Qingfu Zhu ${ }^{1}$ \\ ${ }^{1}$ China Institute of Atomic Energy, P. O. Box 275-45, Beijing 102413,China
}

\begin{abstract}
New advanced reactors require zero-power experiments to validate the correctness of design. In order to ensure sufficient validation strength, certain similarity of physical properties between zero-power experimental reactor and the target reactor is required. Based on sensitivity and uncertainty analysis techniques, three indexes for evaluating the physical similarity between reactor systems were introduced. Based on the Monte Carlo transport program MONK, a coupled similarity analysis program MCS was compiled. The results of MCS were compared with TSUNAMI's and the two programs showed good agreement. Finally, the similarity between the CEFR in MOX fuel loading design and the BFS-119-2 zero-power experiment scheme given by IPPE was calculated by MCS. The results showed that the two systems were very similar to each other, and the physical properties of the CEFR-MOX loading design could be validate by the zero-power experiment.

Key words: similarity analysis; zero-power experiment; validation
\end{abstract}

\section{Introduction}

Zero-power experiment is an indispensable stage in the design of the new tpye reactor[? ].As shown in Figure 1. zero-power experiment determines the validation domain, and the target reactor determines the application domain. It is needed to use the validation domain to infer the application domain, so it is necessary to keep a certain intersection between the validation domain and the application domain. That is, it is necessary to keep a certain similarity in physical properties between zero-power experimental reactor and the target reactor.
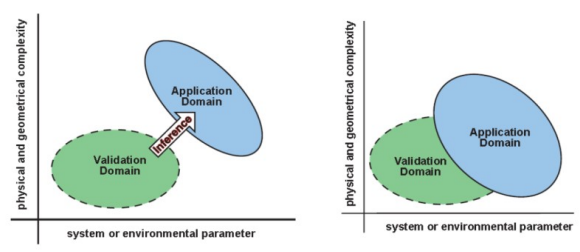

Figure 1: Requirements of validation domain for zeropower experiments.

In order to establish a scientific similarity analysis method between zero-power reactor and target reactor, a method based on sensitivity and uncertainty analysis techniques was proposed[? ]. By using this technique to evaluate the similarity of nuclide sensitivity magnitude and the similarity of $k_{\text {eff }}$ uncertainty sources, the similarity of physical properties between two systems can be quantitatively evaluated.

\footnotetext{
*e-mail: Mailaddressforfirstauthor
}

China Experimental Fast Reactor (CEFR) currently uses uranium dioxide as fuels. In order to study the characteristics of MOX fuels under fast neutron energy spectrum, it is necessary to replace the fuel with MOX fuel. Considering that there has been no actual research in this case, validation by a zero-power experiment is needed .

Based on the Monte Carlo transport program MONK, a coupled similarity analysis program MCS (Monk Coupling Similarity analysis code) was developed. The similarity of physical properties between zero-power experimental reactor and the target reactor is quantitatively evaluated from three aspects: the magnitude of the nuclide sensitivity, the source of the keff uncertainty, and the energy spectrum.

\section{Basic Theory}

The similarity index $C_{k}$ based on the uncertainty analysis is expressed by the definition of the correlation coefficient as:

$$
C_{k}=\frac{S_{i}^{T} C_{a a} S_{j}}{\sqrt{S_{i}^{T} C_{a a} S_{i}} \sqrt{S_{j}^{T} C_{a a} S_{j}}}
$$

Where $S_{i}$ and $S_{j}$ are the sensitivity coefficient vectors of the two systems respectively; $C_{a a}$ is the corresponding relative covariance matrix.

Index $E$ bases on the sensitivity analysis. It uses the angular cosine of the system sensitivity vectors to quantitatively evaluate the similarity and is expressed as:

$$
E=\frac{S_{i}^{T} S_{j}}{\left|S_{i}\right|\left|S_{j}\right|}
$$


The $C_{k}$ and $E$ are similarity indexes that reflect the comprehensive characteristics of the system, and the value ranges both from -1 to 1 .

The index $S$ based on energy spectrum analysis is expressed as:

$$
S=\sum_{g=1}^{G} \frac{s_{g, i}+s_{g, j}}{2} \frac{\min \left(s_{g, i}, s_{g, j}\right)}{\max \left(s_{g, i}, s_{g, j}\right)}
$$

where

$$
S_{g, i}=\frac{\Phi_{g, i}}{\sum \Phi_{g, i}}
$$

is normalized result of energy spectrum, $\Phi_{g, i}$ is neutron flux for the g-th group of i system which calculated by MONK. $S_{g, j}$ is the same. $G$ is the total number of energy groups. The value ranges of $S$ is from 0 to 1

\section{Program Compilation and Verification}

The similarity analysis MCS was compiled using FORTRAN. The calculation process is shown in Figure 2.

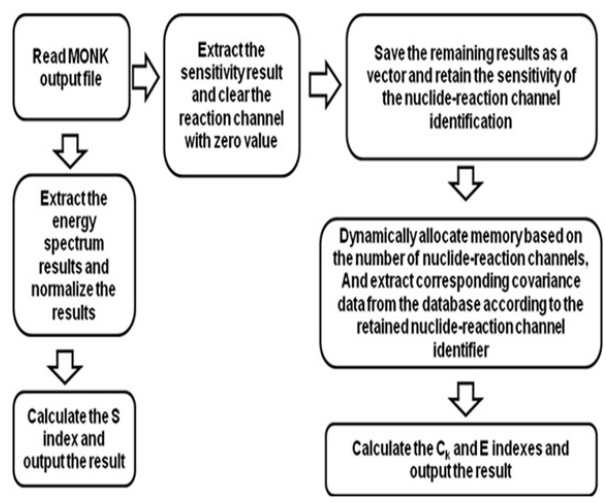

Figure 2: Calculation process of similarity analysis program MCS.

Since the similarity results cannot be verified by experimental data, only the code to code verification method can be selected here. The results of MCS are compared with mature similarity calculation software TSUNAMI's.

23 benchmark experiments covering different fuels, moderator types and energy spectrum types were selected, and similarity results were calculated between them as Figures 3 shown. According to statistics, the relative deviation of similarity index calculated by the MCS program was less than $3 \%$ about $C_{k}$ compared with the results of TSUNAMI, and the relative deviation about $\mathrm{E}$ was less than $6 \%$.

\section{Application in Zero-Power Experimental Validation}

The CEFR-MOX core loading and the corresponding zeropower experiment scheme BFS-119-2 is shown in Fig.5. The $C_{k}$ index of the two reactor systems is calculated to be 0.994 and the $\mathrm{E}$ index is 0.994 . Both of them are close to 1 , that is, the two systems have a high degree of similarity
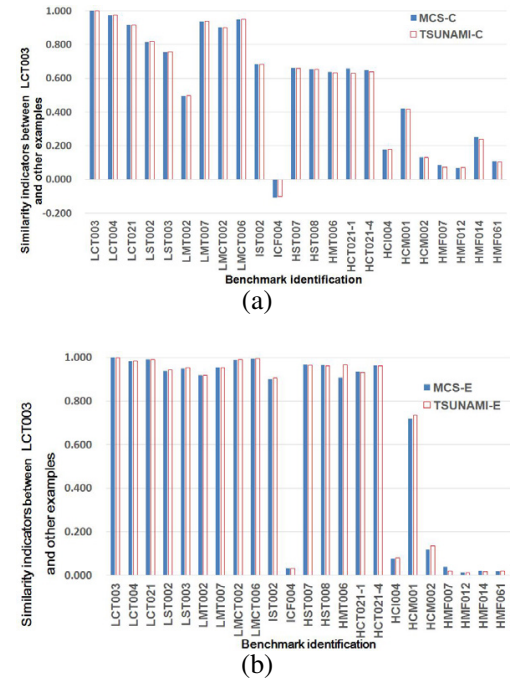

Figure 3: Comparison of similarity indexes between LCT003 and selected cases.

and we can use the experiment results of BFS-119-2 to validate CEFR-MOX-EOC scheme. The calculated $S$ index is 0.920 , and the similarity degree of the energy spectrum is also high.

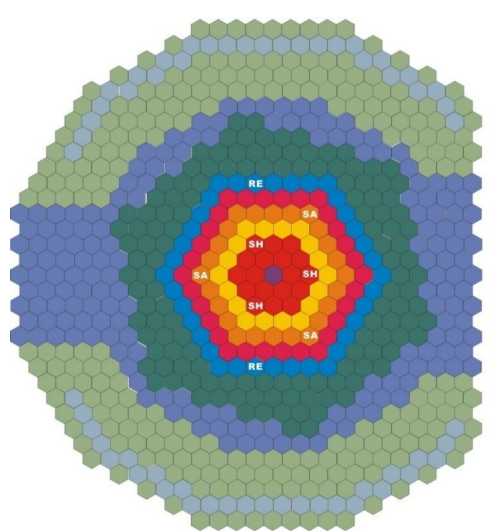

(a)

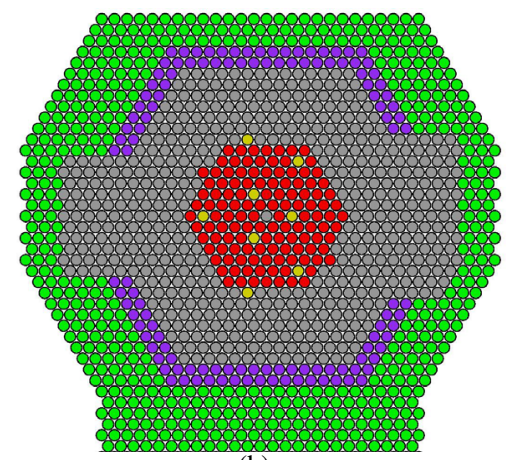

(b)

Figure 4: CEFR-MOX loading (a) and the BFS-119-2 scheme (b). 


\section{Conclusion}

The validation of the target application system via the zero-power experiment involves the similarity of the physical characteristics between the two systems. This paper introduces three indexes for evaluating the physical similarity between systems. Through the similarity calculation, a high similarity between CEFR in MOX loading and the BFS-119-2 scheme is obtained. That is, It can guarantee that the zero-power experiment scheme has sufficient physical property validation strength for CEFRMOX loading design.

\section{References}

[1] Lian Peisheng. Atomic Energy Industry [M]. Beijing: Atomic Energy Press, 2002.

[2] Weisbin C. R., Oblow E. M., Harable J. H., Peele R. W., and Lucius J. L., "Application of Sensitivity and Uncertainty Methodology to Fast Reactor Integral Experiment Analysis," Nucl. Sci. Eng. 1978, 66, 307. 